\title{
Factores asociados a falla virológica en pacientes infectados con VIH que reciben terapia anti-retroviral en un hospital público del Perú
}

\author{
Jorge Alave, Jorge Paz, Elsa González, Miguel Campos, Martin Rodríguez, James Willig y Juan Echevarría
}

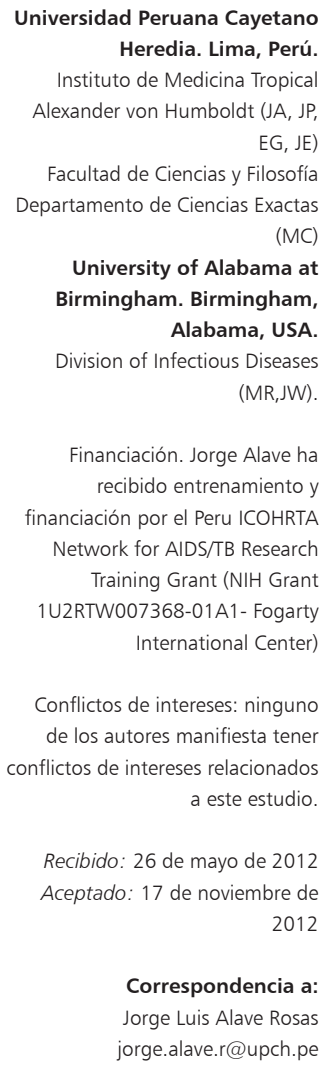

University of Alabama at Birmingham. Birmingham, Alabama, USA.

Division of Infectious Diseases $(M R, J W)$.

Financiación. Jorge Alave ha recibido entrenamiento y financiación por el Peru ICOHRTA Network for AIDS/TB Research

Training Grant (NIH Grant 1U2RTW007368-01A1 - Fogarty International Center)

Conflictos de intereses: ninguno de los autores manifiesta tener conflictos de intereses relacionados a este estudio.

Recibido: 26 de mayo de 2012 Aceptado: 17 de noviembre de 2012

Correspondencia a: Jorge Luis Alave Rosas jorge.alave.r@upch.pe

\section{Introducción}

$\mathrm{E}$ 1 acceso universal a la terapia anti-retroviral de gran actividad (TARGA) ha disminuido la morbimortalidad y mejorado la calidad de vida de las personas infectadas con $\mathrm{VIH} / \mathrm{SIDA}^{1,2}$. El objetivo de la TARGA es lograr niveles indetectables de carga viral y esto se logra en aproximadamente $80 \%$ de los infectados con VIH/SIDA sin exposición previa a anti-retrovirales ("naive") luego de 48 semanas de iniciado el tratamiento 3. Aquellos que no alcanzan este objetivo tienen riesgo de desarrollar resistencia a anti-retrovirales, lo que puede conducir a falla virológica y aparición de enfermedades oportunistas $^{4}$. El valor y el momento de medición de la carga viral con los que se define falla virológica varían entre valores superiores de 48 copias $/ \mathrm{mL}$ y 400 copias $/ \mathrm{mL}$ luego de 24 ó 48 semanas de TARGA, respectivamente ${ }^{5-7}$. Las guías de TARGA recomiendan la monitorización regular de la carga viral para detectar falla virológica. La frecuencia depende del estadio clínico y estabilidad del paciente, pero también de las restricciones locales de los servicios de salud, variando entre 2 y 6 meses $^{6,8}$. En países en desarrollo la frecuencia de obtención de carga viral puede ser incluso menor por dificultades de acceso a la prueba; por esta razón, en los países en vías de desarrollo la falla virológica tiene mayor probabilidad de detectarse tardíamente ${ }^{9}$. Este problema, asociado a otros como las limitadas opciones de anti-retrovirales de segunda línea en países en desarrollo, ensombrece el pronóstico de las personas infectadas con VIH/SIDA con falla virológica.

La falla virológica se ha asociado a factores como terapia anti-retroviral previa, falta de adherencia a la TARGA y resistencia primaria ${ }^{10}$. Estos factores permiten identificar y tratar en forma temprana a estos pacientes y por otro lado implementar estrategias para su control ${ }^{11}$.

En el año 2004, el Ministerio de Salud del Perú empezó la administración gratuita del TARGA según los lineamientos de la Norma Técnica de Tratamiento Antiretroviral de Gran Actividad para pacientes con infección por VIH, aprobada en ese año y actualizada el $2005^{12,13}$. TARGA se define como todo esquema compuesto por tres anti-retrovirales; los regímenes de primera línea consisten en dos inhibidores análogos de transcriptasa reversa (INTR), de preferencia zidovudina y lamivudina 
(alternativamente didanosina, estavudina o abacavir) y un inhibidor no análogo de la transcriptasa reversa (INNTR) como efavirenz o nevirapina. Los inhibidores de proteasa (IP) como lopinavir/ritonavir y atazanavir/ritonavir se reservan para cambio de regímenes por falla virológica o efectos adversos a los regímenes previamente descritos. En la Norma Técnica de TARGA se define falla virológica como carga viral mayor de 400 copias $/ \mathrm{mL}$ al sexto mes de TARGA en caso de resistencia primaria; en caso de resistencia secundaria se define en los pacientes que habiendo alcanzado mediciones de carga viral indetectable presentan valores mayores de 400 copias/mL durante el seguimiento.

En el Perú no se han descrito hasta la fecha las características de las personas infectadas por VIH/SIDA con falla virológica que participan en la Estrategia Sanitaria de Prevención y Control de Infecciones de Transmisión Sexual (ITS)/SIDA según los lineamientos del Ministerio de Salud del Perú ${ }^{12,13}$. Por ello, en este trabajo se examinan las características clínicas y biológicas al ingreso y durante la TARGA de estos pacientes $y$, a través de un análisis multivariado, se plantea determinar cuáles de ellas se asocian de manera independiente con falla virológica en el programa de TARGA de un hospital público del Perú.

\section{Material y Métodos}

\section{Diseño y participantes del estudio}

Se realizó un estudio descriptivo exploratorio y retrospectivo de la información clínica y biológica de los pacientes con infección por VIH/SIDA que participan en la Estrategia Sanitaria de Prevención y Control de Infecciones de Transmisión Sexual (ITS)/SIDA del Hospital Nacional Cayetano Heredia (HNCH), de aquí en adelante, denominado programa VIH/SIDA HNCH. Se incluyeron las fichas de los pacientes sobre 18 años de edad al iniciar TARGA, que contaran con una medición de carga viral luego de 24 semanas de iniciado el tratamiento y que hubiesen sido enrolados entre mayo de 2004 y diciembre de 2009. De un total de 4.320 registros de pacientes infectados por VIH/SIDA enrolados al Programa VIH/ SIDA HNCH, 39,2\% iniciaron TARGA en el período del estudio. Luego de la depuración por los criterios de inclusión restantes, quedaron 1.478 registros de pacientes en TARGA para el análisis, los que fueron seguidos hasta diciembre de 2010.

\section{Definición de variables}

La variable dependiente binomial fue falla virológica, definida como un valor de carga viral superior a 1.000 copias/mL luego de 24 semanas de TARGA. La carga viral se obtuvo utilizando RPC convencional (métodos Amplicor VIH-1 de Roche) en el período de 2004 al
2006, NASBA (NucliSENS VIH-1 de bioMérieux) en el período 2007 al 2009 y RPC en tiempo real (método Cobas TaqMan VIH-1 de Roche) durante el 2010. Esta definición disminuye la posibilidad de incluir individuos que hayan acumulado mutaciones y la confusión con los "blips" (episodios intermitentes de niveles bajos de viremia del VIH). Las variables independientes consideradas fueron la edad, el sexo (masculino o femenino), la conducta sexual (heterosexual u homosexual), el estado clínico al inicio del tratamiento, considerando estadio IV de la clasificación clínica de la OMS (evidencia de SIDA o no), la exposición previa a anti-retrovirales, el tipo de terapia anti-retroviral (basada en INNTR o IP), la carga viral al inicio de TARGA y el recuento de linfocitos T CD4+ (determinado por citometría de flujo) al inicio y un año después de iniciada la TARGA. La carga viral fue categorizada como mayor o menor que 100.000 copias/mL, y el recuento de linfocitos T CD4+ fue categorizado en cuatro niveles: 0-100 céls/mL, 101-200 céls/mL, 201-350 céls/mL y mayor que 350 céls/mL; así mismo, se calculó la ganancia en el recuento de CD4+ al año de iniciada la TARGA. Finalmente, la presencia de infecciones oportunistas y tuberculosis se determinó antes y durante la TARGA. La adherencia a la TARGA, estimada por auto-reporte, también fue incorporada como variable binomial explicativa (cumplimiento del esquema de tratamiento mayor o menor a $95 \%$ ).

\section{Fuente de información}

La información se obtuvo de las fichas de pacientes infectados con VIH/SIDA, sobre 18 años de edad, participantes del Programa VIH/SIDA HNCH En éstas se registra el valor de carga viral y el recuento de linfocitos CD4+ cada vez que se realiza, semestralmente según protocolo pero con marcada variación individual; la aparición de enfermedades oportunistas, eventos adversos asociados a los anti-retrovirales como anemia, exantema, neuropatía periférica, pancreatitis, entre otros; y el nivel de adherencia a la TARGA, considerado óptimo si el porcentaje auto-reportado de toma mensual de anti-retrovirales es superior a 95\%. La calidad de la información de la variable "estadio clínico al inicio de la TARGA" se verificó comparando 100 fichas clínicas del programa VIH/ SIDA HNCH elegidas al azar con la información de la base de datos de la cohorte de VIH/SIDA del Instituto de Medicina Tropical, que incluye participantes del Programa VIH/SIDA HNCH.

\section{Análisis estadístico}

Las variables se describieron utilizando frecuencias y medidas de tendencia central al inicio y durante el tratamiento. Para buscar asociaciones entre falla virológica y las variables estudiadas se realizó un análisis bivariado utilizando la prueba de $\chi^{2}$ o la prueba exacta de Fisher 
para variables categóricas y la prueba de Mann-Whitney para variables continuas. Finalmente, se realizó el análisis de regresión logística multivariado mediante el método de remoción secuencial de variables independientes con menor grado de asociación con la variable dependiente. Se utilizó el programa Epi Info (CDC, versión 3.5.1).

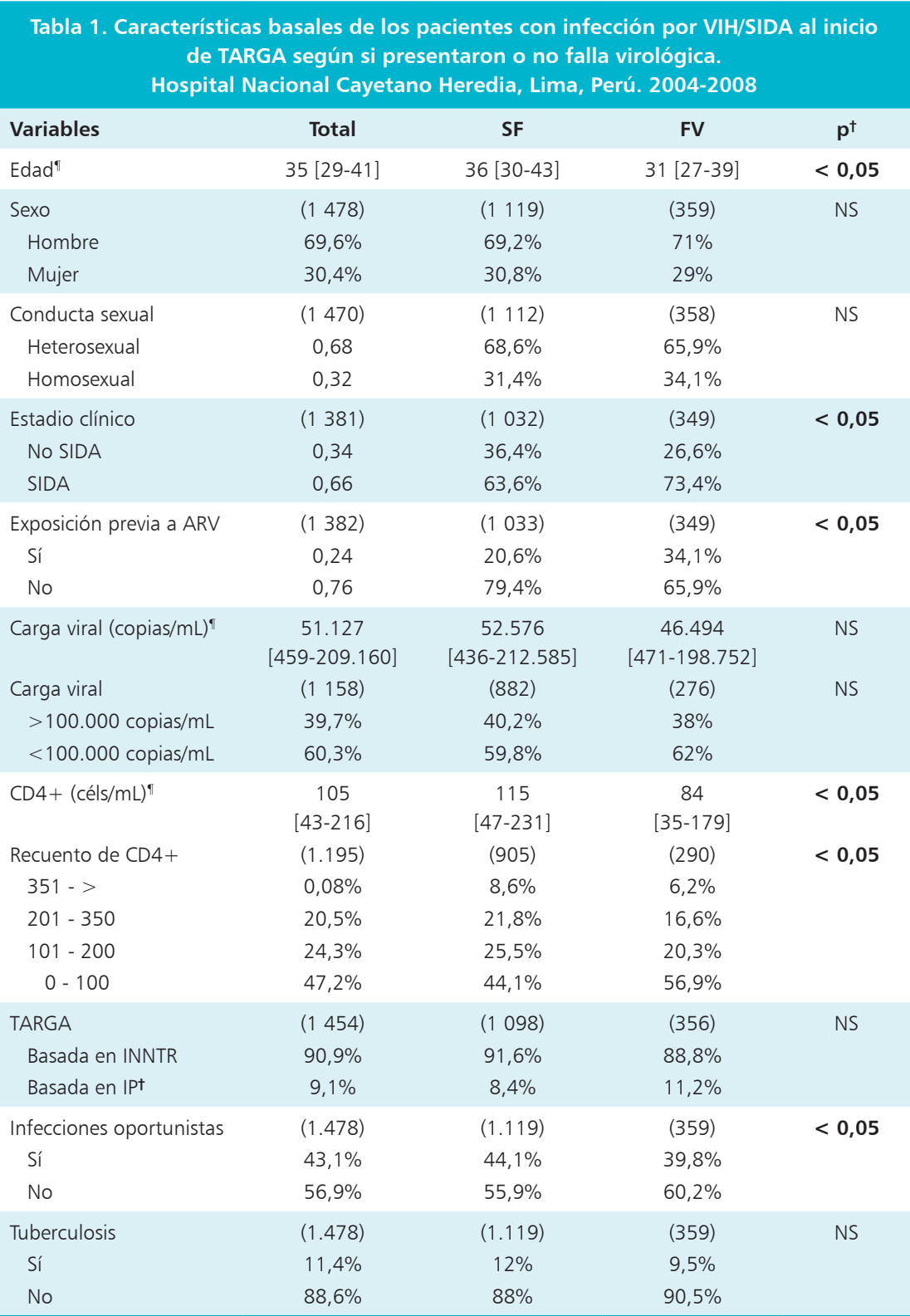

"Se reporta la mediana (acompañada del rango intercuartílico entre corchetes) para las variables continuas y el porcentaje para las categóricas. El valor de $\mathrm{p}$ corresponde a la comparación entre SF y FV. ${ }^{+}$Valor de $p<0,05$ en negrita y NS representa $p>0,05$ (pruebas de $\chi 2$, exacta de Fisher o MannWhitney de acuerdo a si la variable es categórica o continua). TARGA: terapia anti-retroviral de gran actividad. FV: falla virológica. SF: sin falla virológica. ARV: anti-retrovirales. INNTR: inhibidores no análogos de nucleósidos de transcriptasa reversa. IP: inhibidores de proteasa.

\section{Ética}

La utilización de la información se manejó con confidencialidad por los investigadores, sólo ellos tuvieron acceso a la información. Este estudio fue revisado y aprobado por el Comité de Investigación del Instituto de Medicina Tropical Alexander von Humboldt y el Comité de Ética del HNCH, exonerando el uso de consentimiento informado.

\section{Resultados}

Del total de 1.478 registros analizados, 359 (24\%) correspondieron a pacientes que tuvieron falla virológica (FV) y 3,7\% habían fallecido. El mayor porcentaje de fallecimientos ocurrió en el grupo FV (9,5 vs 1,8\% del grupo sin falla virológica [SF], $\mathrm{p}<0,05)$.

Al inicio de la TARGA, la mediana de la edad fue 35 años, (rango intercuartíl [RIQ]: 29-41) y 69,6\% fueron varones. De los casos en los que se registró el estadio clínico, 66\% estuvo en estadio clínico de SIDA según criterios de la OMS. En el grupo FV se observó 10\% más de pacientes en dicho estadio que en el grupo SF. Además, el uso de algún anti-retroviral, en mono o biterapia antes de la TARGA, fue 14\% más frecuente en el grupo FV que en el grupo SF. La mediana del recuento basal de linfocitos T CD4+ fue 105 céls $/ \mathrm{mL}$ y $71 \%$ de ellos presentaron valores inferiores a 201 céls $/ \mathrm{mL}$ (Tabla 1).

La mediana de la ganancia en el recuento de linfocitos CD4+ al año de iniciada la TARGA fue $22 \%$ mayor en el grupo SF que en el grupo FV (+191 céls/mL vs +149 céls/ $\mathrm{mL}, \mathrm{p}<0,05)$. El grupo FV mostró $15,1 \%$ más de casos con niveles inferiores a 350 céls $/ \mathrm{mL}, 2,1 \%$ más de casos de no adherencia y $4 \%$ más de infecciones oportunistas que el grupo SF; estas diferencias tuvieron significancia estadística (Tabla 2). Por otra parte, el cambio de antiretroviral por toxicidad en las primeras 12 semanas de TARGA fue aproximadamente $8 \%$ mayor en el grupo FV que en el grupo SF $(27,3 v s 19,5 \%, \mathrm{p}<0,005)$.

Los resultados de los análisis de asociación se muestran en la Tabla 3. De los factores asociados a falla virológica en el análisis bivariado, sólo persistieron asociados en el análisis multivariado la edad, el antecedente de uso de anti-retrovirales antes del inicio de la TARGA, los niveles de linfocitos T CD4+ inferiores a 100 céls $/ \mathrm{mL}$ al inicio de la TARGA, la adherencia menor de 95\%, el estadio clínico al inicio de la TARGA, el cambio de anti-retrovirales por toxicidad y la aparición de infecciones oportunistas durante la TARGA. Al realizar el mismo análisis en el grupo de pacientes sin antecedente de uso de anti-retrovirales antes del inicio de la TARGA, persistieron asociados en el análisis multivariado la edad (odds ratio[OR]: 0,96; intervalo de confianza [IC] 95\% 0,94-0,98, p < 0,05); la adherencia menor de 95\% (OR: 6,28; IC 95\% 1,83-21,51, 
Tabla 2. Estado de los pacientes con infección por VIH/SIDA luego de 24 semanas de TARGA

según si presentaron o no falla virólogica. Hospital Nacional Cayetano Heredia, Lima, Perú. 2004-2008

\begin{tabular}{|c|c|c|c|c|}
\hline Variables & Total & SF & FV & $p^{\dagger}$ \\
\hline $\begin{array}{l}\text { CD4+ al año" } \\
\text { (céls/mL) }\end{array}$ & $\begin{array}{c}290 \\
{[208-413]}\end{array}$ & $\begin{array}{c}300 \\
{[221-424]}\end{array}$ & $\begin{array}{c}242 \\
{[208-413]}\end{array}$ & $<0,05$ \\
\hline $\begin{array}{l}\text { Recuento de } \\
\text { CD4+ al año } \\
\text { (céls/mL) }\end{array}$ & (1.079) & (857) & (222) & $<0,05$ \\
\hline $351->$ & $35,9 \%$ & $39 \%$ & $23,9 \%$ & \\
\hline $201-350$ & $41,2 \%$ & $41,9 \%$ & $38,7 \%$ & \\
\hline $101-200$ & $18,9 \%$ & $17 \%$ & $26,1 \%$ & \\
\hline $0-100$ & $4 \%$ & $2,1 \%$ & $11,3 \%$ & \\
\hline $\begin{array}{l}\text { Adherencia } \\
\text { mayor del 95\% }\end{array}$ & (1.478) & (1.119) & (359) & $<0,05$ \\
\hline Sí & $98,3 \%$ & $98,8 \%$ & $96,7 \%$ & \\
\hline No & $1,7 \%$ & $1,2 \%$ & $3,3 \%$ & \\
\hline $\begin{array}{l}\text { Cambio de ARV } \\
\text { por toxicidad }\end{array}$ & (1.478) & (1.119) & (359) & $<0,05$ \\
\hline Sí & $21,4 \%$ & $19,5 \%$ & $27,3 \%$ & \\
\hline No & $78,6 \%$ & $80,5 \%$ & $72,7 \%$ & \\
\hline $\begin{array}{l}\text { Infecciones } \\
\text { oportunistas }\end{array}$ & (1 478) & (1 119) & (359) & $<0,05$ \\
\hline Sí & $5,5 \%$ & $4,5 \%$ & $8,9 \%$ & \\
\hline No & $94,5 \%$ & $95,5 \%$ & $91,1 \%$ & \\
\hline Tuberculosis & (1.478) & (1.119) & (359) & NS \\
\hline Sí & $1,6 \%$ & $1,3 \%$ & $2,5 \%$ & \\
\hline No & $98,4 \%$ & $98,7 \%$ & $97,5 \%$ & \\
\hline
\end{tabular}

"Se reporta la mediana (acompañada del rango intercuartílico entre corchetes) para las variables continuas y el porcentaje para las categóricas. El valor de $\mathrm{p}$ corresponde a la comparación entre SF y FV. TValor de $p<0,05$ en negrita y NS representa $p>0,05$ (pruebas de $\chi^{2}$, exacta de Fisher o Mann-Whitney de acuerdo a si la variable es categórica o continua). TARGA: terapia anti-retroviral de gran actividad. FV: falla virológica. SF: sin falla virológica. ARV: anti-retrovirales.

$\mathrm{p}<0,05)$ y la aparición de infecciones oportunistas durante la TARGA (OR:2,2; IC 95\% 1,12-4,33).

\section{Discusión}

La finalidad de este estudio fue describir las características de los pacientes con infección por VIH/SIDA con falla virológica en un total de 1.478 registros obtenidos del programa VIH/SIDA HNCH. La cuarta parte de los registros evaluados cumplió la definición de falla virológica, resultado similar al reportado por Huong y cols. [14]. Entre los pacientes con falla virológica se observó una frecuencia de fallecimientos casi cuatro veces superior a la de los pacientes sin falla virológica. En Latinoamérica, Chile reportó que la tasa de respuesta virológica exitosa entre pacientes reclutados desde octubre de 2001 hasta
Tabla 3. Análisis de regresión logística simple y multivariada para la falla virológica en pacientes con infección por VIH/SIDA que reciben TARGA. Hospital Nacional Cayetano Heredia, Lima, Perú. 2004-2008

\begin{tabular}{|c|c|c|c|c|}
\hline Variables & $\begin{array}{l}\text { OR (IC 95\%) } \\
\text { Simple }\end{array}$ & $\mathbf{p}^{\dagger}$ & $\begin{array}{l}\text { OR (IC 95\%) } \\
\text { Multivariada }\end{array}$ & $p^{\dagger}$ \\
\hline Edad & $0,96(0,94-0,97)$ & $<0,05$ & $0,96(0,94-0,98)$ & $<0,05$ \\
\hline \multicolumn{5}{|l|}{ Estadio clínico } \\
\hline SIDA vs no SIDA & $1,58(1,2-2,06)$ & $<0,05$ & - & - \\
\hline \multicolumn{5}{|c|}{ Exposición previa a ARV } \\
\hline Sí vs No & $1,99(1,52-2,6)$ & $<0,05$ & $3,04(2,13-4,34)$ & $<0,05$ \\
\hline \multicolumn{5}{|c|}{ Recuento de CD4+ al inicio (céls $/ \mathrm{mL}$ ) } \\
\hline $351->$ & 1 (Ref) & & - & - \\
\hline $201-350$ & $1,05(0,58-1,92)$ & NS & - & - \\
\hline $101-200$ & $1,05(0,62-1,79)$ & NS & - & - \\
\hline $0-100$ & $1,59(0,97-2,6)$ & $<0,05$ & - & - \\
\hline \multicolumn{5}{|c|}{ Recuento de CD4+ al año (céls $/ \mathrm{mL}$ ) } \\
\hline $351->$ & 1 (Ref) & & 1 (Ref) & \\
\hline $201-350$ & $1,51(1,04-2,19)$ & $<0,05$ & $1,55(1,04-2,03)$ & $<0,05$ \\
\hline $101-200$ & $2,5 \quad(1,64-3,18)$ & $<0,05$ & $2,85(1,81-4,49)$ & $<0,05$ \\
\hline $0-100$ & $8,75(4,47-17,13)$ & $<0,05$ & $8,98(4,39-18,39)$ & $<0,05$ \\
\hline \multicolumn{5}{|c|}{ Adherencia superior a $95 \%$} \\
\hline Sí vs No & $2,94(1,33-6,51)$ & $<0,05$ & $3,12(0,99-9,84)$ & 0,052 \\
\hline \multicolumn{5}{|c|}{ Cambio de ARV por toxicidad } \\
\hline Sí vs No & $1,55(1,18-2,04)$ & $<0,05$ & $1,47(1,01-2,16)$ & $<0,05$ \\
\hline \multicolumn{5}{|l|}{ IO durante TARGA } \\
\hline Sí vs No & $2,09(1,32-3,32)$ & $<0,05$ & $1,96(1,06-3,61)$ & $<0,05$ \\
\hline \multicolumn{5}{|l|}{ TBC durante TARGA } \\
\hline Sí vs No & $1,89(0,82-4,36)$ & NS & - & - \\
\hline
\end{tabular}

TARGA: terapia anti-retroviral de gran actividad. IO: infecciones oportunistas. TBC: tuberculosis. tValor de $p<0,05$ en negrita y NS representa $p>0,05$.

diciembre de 2007 y seguidos hasta diciembre de 2008 fue de $74 \%{ }^{15}$.

Un nivel de linfocitos T CD4+ inferior a 100 céls/mL al inicio de la TARGA se asoció a falla virológica. Este hallazgo fue encontrado en otras evaluaciones e incluso los niveles de CD4 al inicio y durante la TARGA son utilizados como predictores de falla virológica. DíazGranados y cols. ${ }^{16}$, encontraron que el nivel de CD4 $+<200$ céls/mL y la presencia de enfermedades clasificadas en la categoría $\mathrm{C}$ del CDC estuvieron asociados a resistencia primaria. Robbins y cols. ${ }^{17}$, desarrollaron una escala de predicción clínica para falla virológica utilizando siete predictores clínicos, entre los cuales se consideró recuente de linfocitos T CD4 $+<100$ céls/mL al inicio de la TARGA. Van Oosterhout y cols. ${ }^{18}$, encontraron que un nivel de linfocitos T CD4+ $<200$ céls/mL durante la TARGA se asociaba a falla virológica (definida en este caso como una carga viral $>400$ copias $/ \mathrm{mL}$ ), aunque una escala de predicción de falla virológica basada en este valor no logró sustituir a la medición de carga viral. Hughes y cols. ${ }^{19}$, por 
su parte, asociaron a falla virológica con un incremento reducido de los linfocitos T CD4+ luego de 6 meses de TARGA. A diferencia de los trabajos mencionados, en el presente estudio se evaluaron factores adicionales como infecciones oportunistas, tuberculosis, cambio temprano de anti-retrovirales, entre otros.

El uso de algún anti-retroviral antes de iniciar la TARGA fue otro factor asociado de forma independiente a falla virológica, el cual además podría conducir a la aparición de resistencia viral a los anti-retrovirales ${ }^{20,21}$. Asimismo, otros estudios han encontrado resistencia primaria a los anti-retrovirales en pacientes sin historia de tratamiento previo; ello ha conllevado a considerar o recomendar el uso de pruebas de resistencia antes del inicio de TARGA en lugares con alta prevalencia de este fenómeno ${ }^{6}$. Debido a que en el programa considerado en este estudio no se registra información acerca del tipo de anti-retroviral usado antes del inicio de TARGA, y a que no se realizaron pruebas de resistencia a anti-retrovirales antes del inicio de la TARGA, no podemos describir este factor con mayor detalle.

Otro de los factores con asociación independiente a falla virológica fue la edad, encontrándose que por cada año de disminución de la edad existe aproximadamente 4\% mayor probabilidad de falla virológica. Parenti y cols ${ }^{22}$, encontraron que los pacientes jóvenes (mediana: 38 años; RIQ: 30-69) que presentaron episodios de interrupción de TARGA por más de 48 horas (drug holidays) y depresión tuvieron mayor riesgo de falla virológica. Así mismo, Greenbaum y cols. ${ }^{23}$, describieron que el tiempo a la supresión virológica fue mayor en la población bajo 50 años de edad. Por otra parte, Althoff y cols. ${ }^{24}$, no encontraron supresión virológica significativa con el incremento de la edad. En Colombia, Nagles y cols. ${ }^{25}$, describieron que la no adherencia y la edad inferior a 34 años estuvieron asociadas a falla virológica y DíazGranados y cols. ${ }^{26}$, reportaron falla virológica en pacientes jóvenes (mediana: 34 años; RIQ: 2-61) procedentes de la región oeste de Colombia. Silverberg y cols. ${ }^{27}$, encontraron que a pesar que la población sobre 50 años tuvo mejor respuesta virológica al TARGA, la adherencia fue un factor clave para este resultado y que otros factores como efectos adversos a los anti-retrovirales, co-morbilidades y la senescencia inmune relacionada a la edad pueden influenciar negativamente. Así mismo, Hinkin y cols. ${ }^{28}$, encontraron que a mayor edad las disfunciones neurocognitivas asociadas a la población adulto mayor pueden disminuir la adherencia lo cual conlleva a menor tasa de respuesta virológica al TARGA. Por lo tanto, una edad mayor puede significar un mayor grado de madurez y estabilidad emocional, las cuales contribuirían a una mayor adherencia y a un menor riesgo de hacer falla virológica que la población con menor edad, sin descontar otros factores asociados a la senescencia que pueden contribuir de forma negativa.
Evaluaciones futuras deberán enfocarse en identificar los factores que determinan que una menor edad se asocie a falla virológica en nuestra población.

Otros hallazgos interesantes fueron la asociación de infecciones oportunistas y cambio de anti-retrovirales en las primeras 12 semanas con falla virológica. La aparición de las infecciones oportunistas generalmente es consecuencia de la inmunosupresión provocada por la infección por $\mathrm{VIH}^{29}$, pero infecciones oportunistas como tuberculosis pueden provocar aumento de la replicación del $\mathrm{VIH}^{30}$. En nuestro estudio se encontró que el porcentaje de pacientes con tuberculosis durante la TARGA fue el doble en el grupo FV, pero esto no obtuvo asociación en el análisis multivariado. De otro lado, los efectos adversos potenciales de los anti-retrovirales pueden ocasionar disminución de la adherencia lo cual conlleva a falla virológica ${ }^{31}$. Cesar y cols. ${ }^{32}$, encontraron en siete centros del Caribe y Latinoamérica que una de las razones de cambio de regímenes de anti-retrovirales de primera línea fue la aparición de efectos adversos a estos fármacos. Actualmente se dispone de anti-retrovirales con mejor perfil de efectos adversos. De todos los factores descritos, en el grupo de pacientes sin antecedente de uso de anti-retrovirales antes del inicio de la TARGA, sólo la adherencia, la edad y la aparición de infecciones oportunistas estuvieron asociación con falla virológica.

Este estudio presentó al menos cuatro limitaciones importantes. Primero, las fichas utilizadas no registraron en su totalidad información como nivel socio-económico, estado psicológico, distancia entre el domicilio de los sujetos enrolados y el $\mathrm{HNCH}$, consumo de drogas, entre otros datos que pudieron ser útiles para identificar otros factores asociados a falla virológica. Segundo, debido a que no se realizaron pruebas de resistencia a antiretrovirales, no se investigó la presencia de mutaciones que confirieran al VIH esta resistencia ni se estimó su papel en la aparición de falla virológica. Sin embargo, la resistencia reportada en poblaciones peruanas, que se ha reportado de 1 a 3,3\%, se encuentra por debajo de valores que justificarían un descarte de resistencia antes de iniciar el tratamiento anti-retroviral ${ }^{33,34}$. Tercero, aunque el autoreporte es utilizado para estimar el nivel de adherencia en varios programas de administración de TARGA, las limitaciones propias de este método ${ }^{35}$ podrían explicar el bajo porcentaje de pacientes con nivel de adherencia menor que $95 \%$. Cuarto, no se pudo determinar cuántos de los pacientes que presentaron infecciones oportunistas luego del TARGA, cumplieron criterios de síndrome de inmuno-reconstitución debido al diseño retrospectivo del estudio; es necesario efectuar estudios prospectivos para determinar la prevalencia de este síndrome en nuestra población.

En conclusión, entre los pacientes infectados con VIH/SIDA en seguimiento que reciben TARGA en un 
hospital público del Perú, la falla virológica fue asociada independientemente a jóvenes (aproximadamente $4 \%$ más probabilidad por un año de disminución de la edad), uso de algún anti-retroviral antes de iniciar TARGA, cambio de anti-retrovirales por toxicidad, aparición de infecciones oportunistas durante la TARGA, recuento de linfocitos CD4 $+<100$ céls/mL al inicio de la TARGA, adherencia y estadio clínico; y entre los pacientes infectados con VIH/ SIDA sin antecedentes de uso de anti-retrovirales antes del inicio de la TARGA, la adherencia, la edad y la aparición de infecciones oportunistas estuvieron asociados con falla virológica. La identificación de estos factores en la práctica clínica diaria podría ayudar a reconocer pacientes en riesgo de falla virológica y plantear estrategias para su cuidado. Por otro lado, estudios que evalúen otros factores clínicos, sociodemográficos y biológicos, incluyendo a sujetos con y sin seguimiento durante la TARGA, permitirán definir un mejor perfil de los pacientes con riesgo de hacer falla virológica y diseñar estrategias para el control de este problema en el Perú.

Agradecimientos: Los autores agradecen a Carlos Seas por la revisión metodológica del presente estudio, así también a Daniel Clark, Cristina Guerra y Wilfredo Gonzales por la asesoría en la redacción del presente reporte.

\section{Resumen}

Objetivo: Describir las características clínicas y biológicas de sujetos con falla virológica participantes de la Estrategia Sanitaria de Prevención y Control de Infecciones de Transmisión Sexual (ITS) VIH/SIDA de un hospital público del Perú. Materiales y Métodos: Se realizó un estudio descriptivo exploratorio con datos de sujetos mayores de 18 años que iniciaron terapia antiretroviral de gran actividad (TARGA) entre mayo de 2004 y diciembre de 2009 con al menos un control de carga viral luego de 24 semanas de TARGA. Falla virológica fue definida como un valor de carga viral superior a 1.000 copias/mL luego de 24 semanas de TARGA. Resultados: Se analizaron 1.478 registros de sujetos en TARGA: la mediana de edad fue 35 años (rango intercuartíl [RIQ] 2941) y $69,6 \%$ fueron varones. Se encontró falla virológica en $24 \%$. La proporción de fallecidos fue superior en el grupo con falla virológica $(9,5 \%)$ respecto al grupo sin falla virológica (1,8\%). En el análisis multivariado, la edad, antecedentes de uso de anti-retrovirales antes de iniciar TARGA, cambio de anti-retrovirales por toxicidad, infecciones oportunistas durante TARGA, nivel de linfocitos CD4+ inferior a 100 céls $/ \mathrm{mL}$ al año de inicio de TARGA, adherencia y estadio clínico se asociaron independientemente a falla virológica. En un sub-análisis limitado al grupo de pacientes sin antecedente de uso de anti-retrovirales antes del inicio de la TARGA, se encontró que adherencia, edad y aparición de infecciones oportunistas tuvieron asociación con falla virológica. Conclusión: Este estudio identificó factores asociados a falla virológica. Se necesitan estudios adicionales para evaluar si el uso de estos factores ayudaría a identificar prospectivamente pacientes en riesgo de fallar y al diseño de intervenciones para disminuir este riesgo.

\section{Referencias bibliográficas}

1.- Beard J, Feeley F, Rosen S. Economic and quality of life outcomes of antiretroviral therapy for HIV/AIDS in developing countries: a systematic literature review. AIDS Care 2009; 21: 1343-56.

2.- WHO, UNAIDS, UNICEF. Towards universal access: scaling up priority HIV/AIDS interventions in the health sector. Progress report, Geneva, World Health Organization, 2010. Disponible en: http://whqlibdoc.who.int/ publications/2010/9789241500395_eng.pdf. (accedido 15 de enero de 2012).

3.- Kiertiburanakul S, Sungkanuparph S. Emerging of HIV drug resistance: epidemiology, diagnosis, treatment and prevention. Curr HIV Res 2009; 7: 273-8.

4.- Bartlett JA, Shao JF. Successes, challenges, and limitations of current antiretroviral therapy in low-income and middle-income countries. Lancet Infect Dis 2009; 9: 637-49.

5.- Writing Group, Williams I, Churchill D, Anderson J, Boffito M, Bower M, et al. British
HIV Association guidelines for the treatment of HIV-1-positive adults with antiretroviral therapy 2012. HIV Med 2012; 13 Suppl 2: 1-6.

6.- Panel on Antiretroviral Guidelines for Adults and Adolescents. Guidelines for the use of antiretroviral agents in HIV-1-infected adults and adolescents. Department of Health and Human Services (2012) Disponible en: http:// www.aidsinfo.nih.gov/guidelines. (accedido: 15 de agosto de 2012).

7.- $\quad$ Afani S A, Gallardo O A M. Antiretroviral resistance in human immunodeficiency virus infection. Rev Chilena Infectol 2011; 28: 461-9.

8.- WHO (2010) Antiretroviral therapy for HIV infection in adults and adolescents: Recommendations for a public health approach. Available: http://www.who.int/hiv/pub/arv/ adult2010/en/index.html. (accedido: 15 de agosto de 2012).

9.- Petersen M L, van der Laan M J, Napravnik S, Eron J J, Moore R D, Deeks S G. Long- term consequences of the delay between virologic failure of highly active antiretroviral therapy and regimen modification. AIDS 2008; 22: 2097-106.

10.- Ledergerber B, Egger M, Opravil M, Telenti A, Hirschel B, Battegay M, et al. Clinical progression and virological failure on highly active antiretroviral therapy in HIV-1 patients: a prospective cohort study. The Lancet 1999; 353: 863-8.

11.- Lynen L, Van Griensven, Elliot J. Monitoring for treatment failure in patients on first-line antiretroviral treatment in resource-constrained settings. Curr Opin HIV/SIDA 2010; 5: 1-5.

12.- Ministerio de Salud del Perú. Norma técnica para el tratamiento antiretroviral de gran actividad (TARGA) en adultos infectados por el virus de la inmunodeficiencia humana.2004. Disponible en: http://www.upch.edu.pe/ tropicales/telemedicinatarga/MANUALES $\% 20$ $\% 20$ YGUIAS/NORMA $\% 20$ TECNICA $\% 20$ TARGA(FINAL\%20con\%20anexos).pdf. (accedido: 15 de agosto de 2012).

13.- Ministerio de Salud del Perú. Norma técnica para el tratamiento antiretroviral de gran actividad (TARGA) en adultos infectados 
por el virus de la inmunodeficiencia humana. 2005. Disponible en: http://www.upch.edu.pe/ tropicales/telemedicinatarga/MANUALES\%20 $\% 20$ YGUIAS/NORMA $\% 20$ TECNICA $\% 20$ TARGA(FINAL\%20con\%20anexos).pdf. (accedido: 15 de agosto de 2012).

14.- Huong D T, Bannister W, Phong P T, Kirk O, Peters L. Factors associated with HIV-1 virological failure in an outpatient clinic for HIV-infected people in Haiphong, Vietnam. Int J STD AIDS 2011; 22: 659-64.

15.- Wolf M J, Cortés C P, Shepherd B E, Beltrán C J. Long-term outcomes of a national expanded access program to antiretroviral therapy: The Chilean AIDS Cohort. J Acquir Immune Defic Syndr 2010; 55: 368-74

16.- DíazGranados C A, Mantilla M, Lenis W. Antiviral drug resistance in HIV infected patients in Colombia. Int J Infect Dis 2010; 14: e298-303.

17.- Robbins G K, Johnson K L, Chang Y, Jackson K E, Sax P E, Meigs J B, et al. Predicting virological failure in an HIV clinic. Clin Infect Dis 2010; 50: 779-86.

18.- van Oosterhout J J, Brown L, Weigel R, Kumwenda J J, Mzinganjira D, Saukila N, et al. Diagnosis of antiretroviral therapy failure in Malawi: poor performance of clinical and immunological WHO criteria. Trop Med Int Health 2009; 14: 856-61.

19.- Hughes R A, Sterne J A, Walsh J, Bansi L, Gilson R, Orkin C, et al. Long-term trends in CD4 cell counts and impact of viral failure in individuals starting antiretroviral therapy: UK Collaborative HIV Cohort (CHIC) study. HIV Med 2011; 12: 583-93.

20.- Ségéral O, Limsreng S, Nouhin J, Hak C, Ngin S, De Lavaissière $M$, et al. Short communication: three years follow up of first line antiretroviral therapy in Cambodia: negative impact of prior antiretroviral treatment. AIDS Res Hum Retroviruses 2011; 27: 597-603.

21.- Deeks S G, Hecht F M, Swanson M, Elbeik T, Loftus R, Cohen P T, et al. HIV RNA and CD4 cell count response to protease inhibitor therapy in an urban AIDS clinic: response to both initial and salvage therapy. AIDS 1999; 13: F35-43.

22.- Parienti JJ, Massari V, Descamps D, Vabret A, Bouvet E, Larouzé B, et al. Predictors of virologic failure and resistance in HIVinfected patients treated with nevirapine or efavirenz-based antiretroviral therapy 2004; 38: 1311-6.

23.- Greenbaum A H, Wilson L E, Keruly J C, More R D, Gebo K A. Effects of age and HAART regimen on clinical response in an urban cohort of HIV infected individuals. AIDS 2008; 22: 2331-9 .

24.- Althoff K N, Justice A C, Gange S J, Deeks S G, Saag M S, Silverberg M J, et al. Virologic and immunologic response to HAART, by age and regimen class. AIDS 2010; 24: $2469-79$

25.- Naglés J, Lopera A J, Donado J H, Arbelaez M P, Betancur J, Vélez J A, et al. Factores asociados con el resultado virológico de la terapia antirretroviral altamente activa (HAART) en 340 pacientes naive, Medellín Colombia, 1997-2000. Medicina UPB 2007; 26: 109-25.

26.- DíazGranados C A, Silva A, Bermudez A Roncancio D, Diruggiero P, Mantilla M. Rate and predictors of optimal virologic response to antiretroviral therapy in Colombia. Int J Infect Dis 2007; 11: 531-5.

27.- Silverberg M J, Leyden W, Horberg M A, DeLorenze G N, Klein D, Quesenberry C P Jr. Older age and the response to and tolerability of antiretroviral therapy. Arch Intern Med 2007; 167: 684-91.

28.- Hinkin C H, Hardy D J, Mason K I, Castellón S A, Durvasula R S, Lam M N, et al.
Medication adherence in HIV-infected adults: effect of patient age, cognitive status, and substance abuse. AIDS 2004; 18 Suppl 1: S19-S25.

29.- Oliveira R, Krauss M, Essama-Bibi S, Hofer C, Robert Harris D, Tiraboschi A, et al. Viral load predicts new World Health Organization stage 3 and 4 events in infected children receiving highly active antiretroviral therapy, independent of CD4 T lymphocyte value. Clin Infect Dis 2010; 51: 1325-33.

30.- Pawlowsky A, Jansson M, Skold M, Rottenberg M E, Kallenius G. Tuberculosis and HIV co-infection. PLoS Pathog 2012; 8: e1002464.

31.- Faucher JF, Challier B, Chirouze C, Drobacheff C, Fischer P, Lanq JM, et al. Predictive factors of virological response to primary antiretroviral treatment. Presse Med 2004; 33: 310-5.

32.- Cesar C, Shepherd B E, Krolewiecki A J, Fink V I, Schechter M, Tuboi S H, et al. Rates and reasons for early change of first HAART in HIV-1-infected patients in 7 sites throughout the Caribbean and Latin America. PLoS One 2010; 5: e10490.

33.- Lama J R, Sánchez J, Suárez L, Caballero P, Laguna A, Sánchez J L, et al. Linking HIV and antiretroviral drug resistance surveillance in Peru: a model for a third-generation HIV sentinel surveillance. J Acquir Immune Defic Syndr 2006; 42: 501-5.

34. Soria J, Bull M, Mitchell C, La Rosa A, Dross S, Kraft K, et al. Transmitted HIV resistance to first-line antiretroviral therapy in Lima, Peru. AIDS Res Hum Retroviruses 2012; 28: 333-8

35.- Nieuwkerk P T, Oort F J. Self-reported adherence to antiretroviral therapy for HIV-1 infection and virologic treatment response: a meta-analysis. J Acquir Immune Defic Syndr 2005; 38: 445-8. 